Écrire

l'histoire

\section{Écrire l'histoire}

Histoire, Littérature, Esthétique

$11 \mid 2013$

Présent (1)

\title{
Notes sur la counterfactual history
}

Robert W. Fogel, Raymond Aron et quelques autres

\section{Éric Vial}

\section{(2) OpenEdition}

\section{Journals}

Édition électronique

URL : http://journals.openedition.org/elh/316

DOI : 10.4000/elh.316

ISSN : 2492-7457

Éditeur

CNRS Éditions

\section{Édition imprimée}

Date de publication : 15 mai 2013

Pagination : 113-121

ISBN : 978-2-35698-058-8

ISSN : 1967-7499

Référence électronique

Éric Vial, « Notes sur la counterfactual history», Écrire l'histoire [En ligne], 11 | 2013, mis en ligne le 05 mai 2016, consulté le 23 septembre 2020. URL : http://journals.openedition.org/elh/316 ; DOI : https:// doi.org/10.4000/elh.316 


\section{Notes sur la counterfactual history}

\section{Robert W. Fogel, Raymond Aron et quelques autres}

$\mathbf{E}_{\mathrm{N}}$ apparence, les historiens rejettent une histoire écrite avec des « si ». Leur corporation suit le sens commun pour lequel une telle démarche permettrait de dire n’importe quoi et de mettre Paris en bouteille: «L'histoire est assez difficile comme elle l'est - comme elle l'est réellement sans s'encombrer de questions impossibles ${ }^{1}$. " Dans ces conditions, on comprend que, en 1964, le livre de Robert W. Fogel Railroads and American Economic Growth. Essays in Econometric History ${ }^{2}$ ait causé un petit scandale, mais cette counterfactual history est en réalité beaucoup plus pratiquée qu'il ne l'est avoué, même si c'est de façon fugace et quasi clandestine; et elle a la vertu de faire réfléchir chacun sur sa pratique, ses a priori, sa vision de l'histoire.

Utilisant l'économétrie, Fogel présentait ce qu'aurait été l'évolution des États-Unis au $\mathrm{XIX}^{\mathrm{e}}$ siècle sans le chemin de fer. On a parlé, côté anglo-saxon, d'un auteur "frappé d'anathème par certains historiens », d'une « ancienne forme d'erreur récemment élevée au rang de méthode », d'« anti-histoire », de « non-histoire », de « quasi-histoire », d' « histoire-fiction », « c'està-dire nullement de l'histoire véritable ». Fritz Redlich, le critique hostile le plus cité, évoquait

Éric Vial, université de Cergy-Pontoise, CICG - Civilisations et identités culturelles comparées.

1. David H. Fischer, cité par Jean Heffer, « Le dossier de la question », dans Ralph Andreano (dir.), La Nouvelle Histoire économique. Exposés de méthodologie [Explorations in Entrepreneurial history, 1969], trad. de l'anglais par Roger Gilles, Gallimard (Bibliothèque des histoires), 1977, p. 69-70.

2. Robert W. Fogel, Railroads and American Economic Growth. Essays in Econometric History, Londres/Baltimore, Johns Hopkins Press, 1964, p. 219. 
des « travaux [qu'il ne saurait] d'aucune façon accepter comme de l'histoire économique » au nom de la distinction entre hypothèses et figments, créations de l'esprit non vérifiables ${ }^{3}$. En France, Jean Heffer a noté que « cette technique est celle qui a suscité les plus fortes oppositions chez les historiens traditionnels » et Pierre Chaunu exécuta Fogel, sans même le nommer, d'un « on a atteint l'absurde ${ }^{4} »$.

Mais Fogel étant aussi marginal que peut l'être le premier historien américain élu à l'Académie nationale des sciences, de surcroît « Nobel » d'économie (1993), le tollé s'est vite calmé aux ÉtatsUnis, où les critiques étaient en fait loin de se focaliser sur la counterfactual history, lui reprochant plutôt de ne pas remonter assez avant 1860, d'escamoter ouvertement des effets dérivés ou multiplicateurs, de raisonner sur la seule agriculture faute de tout calculer, de sous-estimer les facteurs politiques, sociaux ou psychologiques du développement urbain, bref, de ne pas examiner toutes les variables. Plus des interrogations techniques: efficacité des canaux, effets du gel, surcoûts cachés, etc. S'y ajoutaient les réactions à sa thèse de fond, l'inutilité du rail pour la révolution industrielle, et à son ton ou à sa volonté d'en découdre avec ses aînés. On a par ailleurs ironisé sur l'allergie de certains de ses critiques à tout chiffre autre qu'un numéro de page ${ }^{5}$. Il est possible d'ajouter que Fogel a pu construire un marteau-pilon pour

3. Julius Robin, compte rendu, Technology and culture, 1967, p. 228; Lance B. Davis, «"Et ce ne sera plus jamais de la littérature”. Une critique de la nouvelle histoire économique », dans Ralph Andreano (dir.), op. cit., p. 198; David H. Fischer, Historians' Fallacies. Toward a Logic of Historical Thought, Londres, Routledge and Kegan Paul, 1971, p. 15, 195-196; Jean Heffer, « Une histoire scientifique: la nouvelle histoire économique », Annales. ESC, 1977, p. 825; George S. Murphy, « On Counterfactual Propositions », History and Theory, Beiheft 9. Studies in Quantitative History and the Logic of the Social Sciences, Middletown, Wesleyan University Press, 1969, p. 17; Fritz Redlich, «Potentialités et traquenards en histoire économique », dans Ralph Andreano, op. cit., p. 213-216; id., "New" and Traditional Approaches to Economic History and Their Interdependence », Journal of Economic History $(\mathcal{J E H}), 1965$, p. 486; cité aussi dans Robert W. Fogel, « The New Economic History. Its Findings and Methods », Economic History Review (EHR), 1966, p. 643.

4. Jean Heffer, «La Nouvelle Histoire économique », Bulletin du Centre d’histoire économique et sociale de la région lyonnaise, 1976, $\mathrm{n}^{\circ} 2$, p. 66, ou id., «Le dossier... », p. 69; Pierre Chaunu, «L'économie. Dépassement et prospective », dans Jacques Le Goff, Pierre Nora (dir.), Faire de l'Histoire, t. 2, Nouvelles approches, Gallimard, 1974, p. 63.

5. Alfred D. Chandler Jr., « Commentaire de l'exposé du professeur Conrad », dans Ralph Andreano, op. cit., p. 287 ; Edward C. Kirkland, compte rendu, American Historical Review (AHR), 1967, p. 1494-1495; Meghnad Desai, « Some Issues in Econometric History », EHR, 1968, p. 10-11, 16; Fritz Redlich, " "New”... », p. 487 ; John Tosh, The Pursuit of History. Aims, Methods and New Directions On the Study of Modern History, Londres/New York, Longman, 1984, p. 165; Thomas C. Cochran, «Economic History, Old and New », AHR, 1969, p. 1567-1568; Julius Robin, compte rendu, p. 228, 231, 233; William N. Parker, «From Old to New to Old in Economic History », fEH, 1971, p. 7; J. D. Gould, « Hypothetical History », EHR, 1969, p. 205; Richard J. Evans, In Defence of History, Londres, Granta Books, 1997, p. 41 ; Paul A. David, « Transport In- 
écraser une noisette: la non-nécessité du rail est démontrée dans son livre en une ligne où il note que l'Angleterre s'est avancée dans la révolution industrielle bien avant l'apparition du chemin de fer ${ }^{6} \ldots$ En tout cas, les discussions ont peu porté sur la méthode contrefactuelle.

Par ailleurs, Fritz Redlich, en particulier, a été plus nuancé que ne l'ont dit certains, qui ont admis l'avoir lu trop vite ${ }^{7}$. Pour lui, « avec l'aide d'hypothèses nous gagnons du savoir, avec celle d'un figment nous obtenons un outil pour l'acquisition du savoir ». Ce n'est pas une condamnation. Il ajoute:

Si la recherche historique à l'aide d'hypothèses est quelque chose de plus qu'une marotte, je la classifierais dans la construction de modèles, c'est-à-dire l'élaboration d'instruments en vue d'une recherche empirique réaliste. Son utilité à cette fin justifierait l'effort entrepris. L'utilité pratique des fictions dans la science sociale, par opposition à la recherche historique, est en effet démontrée.

Il ne s'« élève pas contre ce genre de recherche en lui-même, pas plus [qu'il] ne le considère comme étant sans valeur. [ll veut] seulement qu'on admette qu'il fait partie intégrante des sciences sociales, et [il tient] à insister sur son caractère d'instrument dans la mesure où il s'agit de l'histoire ». Selon lui, la démarche est intéressante mais non historique, les changements à travers le temps ou provoqués par les hommes du passé relevant seuls de l'histoire. D'où l'idée de « questions antéhistoriques », préalables ${ }^{8}$. En gros, Redlich fait de la counterfactual history une sorte de science auxiliaire, ce qui ne saurait être infamant.

Cette même counterfactual history s'est développée hors du quantifiable économétrique, jusqu'en histoire politique. Ainsi Stuart Bruchey a-t-il proposé, comme exercice mental, de s’interroger sur un New Deal sans Roosevelt, afin de «se concentrer sur les pistes de développement qu'auraient pu prendre d'autres tendances et d'autres forces agissantes ", en précisant que « ce n'est pas un "savoir scientifique" que procure l'emploi du contrefactuel, mais bien une conscience affinée des multiples variables se rapportant à l'objet de la recherche ${ }^{9}{ }$. De même,

novation and Economic Growth. Professor Fogel on and off the Rails », EHR, 1969, p. 511; Lance B. Davis, «"Et ce ne sera plus jamais de la littérature"... », p. 182; id., «Professor Fogel and The New Economic History », EHR, 1966, p. 660;

Alexander Gerschenkron, « The Discipline and I », fEH, 1967, p. 447, 455; Robert W. Fogel, « The Specification Problem in Economic History », fEH, 1967, p. 283; George S. Murphy, « On Counterfactual Propositions », p. 17.

6. Robert W. Fogel, Railroads..., p. 235.

7. Alfred H. Conrad, « L'économétrie et l'histoire du sud des États-Unis », dans Ralph Andreano, op. cit., p. 257.

8. Fritz Redlich, «"New"... », p. 484 (cette traduction et la suivante sont les nôtres); id., « Potentialités et traquenards... », p. 215-216, 218-219.

9. Stuart Bruchey, « Commentaire de l'exposé du professeur Conrad », dans Ralph Andreano, op. cit., p. 273. 
Richard Hofstadter s'est demandé ce qui aurait changé si un protestant avait été élu à place de Hoover en 1928, tel autre comment aurait évolué l'esclavage sans la guerre de Sécession ${ }^{10} \ldots$ On peut y voir un effet de la culture anglo-saxonne, plus ouverte à l'imaginaire que la nôtre, et où Arnold Toynbee a mêlé à ses essais «normaux » des textes rêvant l'évolution du monde après une liquidation précoce d'Alexandre par Philippe de Macédoine, sans Empire romain, ou au contraire un État-monde après le règne prolongé du même Alexandre ${ }^{11}$.

Cela dit, la counterfactual history économétrique n'a guère été plus qu'un feu de paille aux ÉtatsUnis, alors qu'elle a pu être bien reçue en France. Ainsi, Maurice Lévy-Leboyer s'est interrogé sur la validité des indices de Fogel et lui a reproché de trop faire appel au « toutes choses égales par ailleurs » en oubliant les interactions, mais c'était inviter à approfondir la démarche et non la rejeter. Il a aussi suggéré ${ }^{12}$ d'imaginer une croissance non pas privée d'un facteur mais débarrassée d'un obstacle, d'en envisager les effets sur la technologie ou les mouvements de population, etc. Heffer, lui, a noté que « l'analyse contrefactuelle est [...] un élément central dans l'explication et non ce monstre fictif qu'ont décrit des historiens » et que « les réticences devant son emploi devraient tomber, au moins chez ceux qui ne sont pas éblouis d'une révérence superstitieuse pour l'événement ${ }^{13}{ }^{\prime}$.

Par ailleurs, dans des travaux très divers, on trouve des exemples d'usage modéré de cette démarche ${ }^{14}$. Ainsi, Yves Lequin notait qu'on pourrait imaginer, sans la crise agricole de la fin du XIX ${ }^{\mathrm{e}}$ siècle, une industrie française en décollage mais avec un pourcentage d'ouvriers en baisse dans la population; Mathias Bernard, dans une synthèse sur la France de 1958 à 1981, évoque le choix de René Coty en 1958:

Que se serait-il passé s'il avait tenté une des deux autres options qui lui étaient offertes, c'est-à-dire l'entente directe avec le général Salan et le comité de salut public, ou bien la résistance frontale à la menace de coup d'État par la mobilisation de toutes les forces politiques (y compris le PCF) et des syndicats? Le pragmatisme du dernier président de la IV République a facilité la tâche de son successeur, qui a pu fonder son régime sur le thème du retour à l'ordre républicain et non sur le souvenir d'un coup d'État.

10. Cf. George Green, « Commentaire des deux exposés précédents », dans Ralph Andreano, op. cit., p. 231.

11. Arnold Toynbee, Some Problems of Greek History, Londres, Oxford University Press, 1969, p. 418-420, 440, 484-486.

12. Maurice Lévy-Leboyer, « Chemins de fer et croissance économique: l'exemple américain », Annales. ESC, 1966, p. 637; id., «La "New Economic History” », Annales. ESC, 1969, p. 1057, 1062, 1064.

13. Jean Heffer, «Une histoire... », p. 832.

14. On pourrait, au moins par jeu, remonter jusqu'à Hérodote qui imagine ce qui serait arrivé si « devant le danger qui les menaçait, les Athéniens terrifiés avaient abandonné leur patrie, ou si, au lieu de l'abandonner, ils étaient restés chez eux et s'étaient soumis à Xerxès ». "L'Enquête », Livre VII, 139, dans Hérodote-Thucydide, CEuvres complètes, Gallimard (Bibliothèque de la Pléiade), 1979, p. 508-509. 
On peut remonter à Georges Vedel imaginant, en 1964, le président issu des institutions de 1958 si un attentat contre le général de Gaulle avait réussi avant que le président de la République doive être élu par tous, ou à René Grousset esquissant, en 1948, d'abord une histoire sans Napoléon, avec Hoche en Washington français et où «quelque III ${ }^{\mathrm{e}}$ République serait ainsi directement sortie de la première. Quelle économie de discordes civiles, de convulsions et de temps! »; puis à l'inverse un succès monarchien et Louis XVIII anticipant de vingt ans la Charte de 1830, enfin Bonaparte vainqueur à Saint-Jean-d'Acre et fondant un empire en Asie sur les traces d'Alexandre ${ }^{15}$. Bref, l'histoire non advenue n'est pas tout à fait taboue en France.

Reste à se demander à quoi elle sert. Il faut écarter un rêve: l'histoire devenant une science expérimentale. C'est peut-être le grand regret refoulé de la corporation, formalisé par Daniel S. Milo:

L'historien ne dispose pas de son matériau comme le biologiste des bactéries, l'écrivain des mots. Jamais il ne pourra ajouter Frédéric II au XVII siècle, pour vérifier l'applicabilité du modèle Elias au livre de Kantorowicz, jamais il ne pourra arracher les chemins de fer de l'histoire américaine du XIX siècle, jamais il n'aura entre ses mains une étude d'urbanisme du Paris de 1987 écrite par le baron Haussmann. ${ }^{16}$

On reste du côté de l'exercice mental, non de l'administration de la preuve.

Mais l'exercice a autant de vertus heuristiques que la métaphore ou l'analogie. Il aide à se poser des questions, à s'interroger sur le poids relatif des causes ${ }^{17}$, etc. Il permet d'isoler un facteur, de préciser le poids implicite qu'on lui attribuait. Il peut aider à déconstruire une causalité qui n'est en fait qu'une coïncidence, à mettre en lumière des mécanismes moins aveuglants mais plus réels, à se mettre à la place des acteurs. Raymond Aron notait que « si la politique, c'est la décision face à une situation actuelle, l'histoire, elle, est la considération rétrospective des décisions prises par d'autres, et quelquefois l'effort pour se replacer au moment de la décision, pour spéculer sur les possibles qui ne sont pas devenus réels. Quand nous spéculons sur ce que nous devons faire, nous partons toujours de l'idée - et nous en sommes toujours convaincus - que nous pouvons faire $\mathrm{A}$ ou $\mathrm{B}$, et que le choix de $\mathrm{A}$ ou

15. Yves Lequin, Les Ouvriers de la région lyonnaise. La formation de la classe ouvrière régionale, t. 1, PUL, 1977; Mathias Bernard, La France de mai 1958 à mai 1981. La grande mutation, Libraire générale française (Livre de Poche), 2003, p. 25; Georges Vedel, «Vers le régime présidentiel? », Revue française de science politique, 1964, p. 27 ; René Grousset, Figures de proue, rééd., Balland, 1992, p. 203, 222-223, 303-304.

16. Daniel S. Milo, « Pour une histoire expérimentale, ou le gai savoir », dans Daniel S. Milo et Alain Boureau (dir.), Alter Histoire. Essais d'histoire expérimentale, Belles Lettres, 1991, p. 21.

17. Alexander Demandt, Ungeschehene Geschichte, Göttingen, Vandenhoeck Ruprecht, 1986, p. 16-37, passim; David H. Fischer, Historians' Fallacies..., p. 16. 
de $\mathrm{B}$ aura des conséquences ${ }^{18} »$. Dans les facteurs déterminant une action intervient l'idée que l'acteur se fait des suites d'un autre choix. C'est ce que suggérait l'esquisse des possibles en mai 1958. De même, demander qui aurait remplacé de Gaulle en 1962 aide à retrouver en partie le raisonnement de ce dernier, sans doute peu enthousiaste à l'idée d'avoir Antoine Pinay pour successeur. Enfin, les projets non réalisés ont leur importance, ne serait-ce qu'à cause des réactions suscitées, par exemple dans les relations internationales; d'où la légitimité d'une interrogation sur leurs résultats potentiels, qu'il s'agisse d'une élection de François I ${ }^{\text {er }}$ à la tête du Saint Empire ou des multiples partages des Balkans avant $1914^{19}$. Ce qui a été imaginé, craint ou souhaité est objet d'histoire comme toute réalité.

La counterfactual history a d'autres vertus, qu'il s'agisse de réfléchir sur la pratique des historiens, sur les conceptions en matière de causalité ou sur la nature de l'histoire entre fatalité et liberté. Dans la réalité, la démarche est fréquente mais quasi clandestine.
Même si l'historien ne doit pas se muer en juge de la vallée de Josaphat, dire qu'une action est une faute suppose une comparaison avec ce qui aurait pu être, et tout jugement sur un fait suppose une idée de ce qui serait advenu sans lui. Fritz Redlich voit du contrefactuel clandestin derrière des affirmations sur le caractère « indispensable » de ceci ou de cela. Fogel, lui, souligne qu'il ne s'agit pas de «mettre en circulation de $[\mathrm{s}]$ propositions contrefactuelles nouvelles », mais d'« évaluer la validité empirique de ces propositions qui étaient déjà explicites ou implicites dans la littérature existante ${ }^{20} »$. Il s'agit donc de rendre explicite un discours, et Heffer a noté que « les critiques les plus vives » contre l'histoire contrefactuelle viennent des « historiens traditionnels, alors qu'eux-mêmes n'hésitent pas à en abuser, mais sans le savoir », quitte à nuancer « certaines affirmations exagérées tendant à proclamer qu'il y avait un conditionnel irréel derrière chaque proposition causale ${ }^{21} »-$ cette prudence de la fin des années 1970 a été ensuite moins nécessaire, et on a pu lire en 1990 dans les Annales qu'« il y a souvent de l'inconditionnel irréel implicite dans l'énoncé des jugements historiques ${ }^{22} »$.

18. Raymond Aron, Leçons sur l'histoire [1989], Éd. de Fallois (Livre de Poche), 2007, p. 332.

19. Régine Pernoud, La Formation de la France, PUF, $3^{3}$ éd., 1965 ; Jean-François Ségard, Éric Vial, Nations, Nationalismes, Nationalités en Europe de 1850 à 1920. Atlas, Ellipses, 1996.

20. Thomas G. Cochran, « Economic History, Old and New », p. 1568; Lance B. Davis, « Professor... », p. 657 ; id., « "Et ce ne sera plus jamais de la littérature"... », p. 195, 197 ; Robert W. Fogel, Railroads..., p. 264-265; id., «The Specification... », p. 285; id., « The New... », p. 655; Fritz Redlich, « Potentialités et traquenards... », p. 217; Alfred H. Conrad, « L'économétrie et l'histoire... », p. 287; George Green, « Commentaire des deux exposés... », p. 231; George S. Murphy, « On Counterfactual Propositions », p. 18; Alexander Gerschenkron, « The Discipline and I », p. 454.

21. Jean Heffer, « Une histoire...», p. 832; id., « Le dossier... », p. 70.

22. Daniel S. Milo, « Pour une histoire expérimentale, ou la gaie histoire », Annales. ESC, 1990, p. 726. 
Au-delà de ce problème de méthode, la counterfactual history pousse à s'interroger sur les causalités et le hasard. On peut partir du nez de Cléopâtre, ou, également chez Pascal, de Cromwell qui « allait ravager toute la chrétienté; la famille royale était perdue, et la sienne à jamais puissante, sans un petit grain de sable qui se mit dans son uretère. Rome même allait trembler sous lui. Mais ce petit gravier s'étant mis là, il est mort, sa famille abaissée, tout en paix, et le roi rétabli ${ }^{23}$ ». Le principe du « petites causes, grands effets » peut fasciner ou irriter. Porté à l'extrême, il débouche sur une folie logique des mécanismes et des enchaînements, proche du burlesque, qu'avec Voltaire et Frédéric II on remonte de la récupération de Naples par l'Espagne à la paix d'Utrecht, puis à la chute des whigs, et de là à une brouille entre Anne d'Angleterre et Lady Malborough pour une commande de gants, ou, avec Churchill, des 250000 morts de la guerre gréco-turque à la défaite de Venizélos dans une élection nécessitée par la mort du roi Alexandre après la morsure d'un singe défendant sa guenon contre le chien du monarque ${ }^{24}$. À l'inverse, au nom du sérieux, on peut en appeler à Montesquieu: « Si le hasard d'une bataille, c'est-à-dire une cause particulière, a ruiné un État, il y avait une cause générale qui faisait que cet État devait périr par une seule bataille ${ }^{25}$ », et on imaginera qu'une victoire de Napoléon à Waterloo n'aurait changé qu'un nom de gare à Londres - encore que Wellington ait pu être à bout de forces et près d'abandonner la partie.

Le jeu de la nécessité et de la contingence engage par ailleurs une vision du monde. De fait, il est facile de dénoncer une conception relevant du proverbial petit bout de la lorgnette, et de mettre en avant des événements que la tradition suppose déterminants et qui ne sont que l'aboutissement d'un long cheminement. Mais cela fonctionne moins bien à propos de situations de crise, ou des débuts d'un processus. On peut certes plaider que les effets d'un accident sont limités ${ }^{26}$, que les causes profondes reprennent le dessus, et que le prétexte - coup de feu à Sarajevo en juin 1914 ou représentation au théâtre de la Monnaie à Bruxelles en août 1830 - peut être remplacé par un autre pour qu'éclatent la Grande Guerre ou la révolution d'où naît la Belgique ${ }^{27}$ - mais on peut aussi douter que l'histoire « repasse les plats » si libéralement et qu'un « prétexte » plus tardif rencontre une conjoncture aussi favorable. Là encore, l'historien peut s'interroger sur ce qu'il croit essentiel ou

23. Blaise Pascal, Pensées, dans Euvres complètes, Gallimard (Bibliothèque de la Pléiade), 1954, p. 1147-1148.

24. Cf. Jean Stengers, Vertige de l'historien. Les histoires au risque du hasard, Les Empêcheurs de penser en rond, 1998, p. 24-26, 33-34, 149.

25. Montesquieu, Considérations sur les causes de la grandeur des Romains et de leur décadence, Garnier-Flammarion, 1968, p. 145.

26. Richard J. Evans, In Defence of History, p. 132.

27. Pierre Salmon, Histoire et Critique, Bruxelles, Université de Bruxelles, 1976, p. 160-161. 
anecdotique. Tout est par ailleurs question d'échelle. On peut ainsi penser que le rythme des découvertes de gisements miniers ne doit rien au hasard, et répond à la demande ou à la nécessité, car il obéit à des lois globales ${ }^{28}$, mais selon que les découvertes se font là ou ailleurs, si la situation économique mondiale n'est guère modifiée, celle des pays l'est beaucoup - Marc Bloch disait déjà qu'il aurait pu ne pas y avoir d'or en Amérique ${ }^{29}$. Sur le plan politique, Edward H. Carr note à propos de la mort de Lénine que même «si on estime que, sur la longue durée, les choses seraient revenues au même, il y a un temps court qui est important, et qui fait une différence considérable pour énormément de gens ${ }^{30} »$. D’une certaine façon, Marx, dont la pensée est réputée incompatible avec ces perspectives au point que l'uchronie, contrepartie littéraire de la counterfactual history, semble avoir été bannie de feu l'URSS et de ses satellites ${ }^{31}$, ne dit rien d'autre:

Les hasards fortuits rentrent naturellement dans la marche générale de l'évolution et se trouvent compensés par d'autres hasards. Mais l'accélération ou le ralentisse- ment du mouvement dépendent beaucoup de semblables « hasards », parmi lesquels figure aussi le « hasard» du caractère des chefs appelés les premiers à conduire le mouvement. ${ }^{32}$

Charles Renouvier aurait pu y souscrire, car son Uchronie accélérait l'histoire du monde, effaçait le Moyen Âge, arrivait directement à l'équivalent de la Renaissance grâce à Avidius Cassius et à des réformes dont l'idée existait bien dès la Rome antique ${ }^{33}$. Les accélérations plus modestes de Grousset, déjà évoquées, vont dans le même sens. Mais on peut y voir une conséquence des limites de l'imagination et de la relative facilité à imaginer une histoire reconvergeant avec la nôtre plutôt que s'en éloignant de plus en plus...

$\mathrm{Au}$ total, la counterfactual history illustre un mouvement, auquel elle participe, visant à « défataliser » l'histoire ${ }^{34}$ : notre passé figé a été du futur ouvert. On retrouve Aron, qui, à partir de l'idée d'une victoire allemande sur la Marne en 1914, invoquait une histoire événementielle qui « a pour fin, pour signification et pour intérêt

28. Geoffrey Blainey, «A Theory of Mineral Discovery. Australia in the Nineteenth Century », EHR, 1970, p. $298,313$.

29. Marc Bloch, «Le salaire et les fluctuations économiques à longue période », Revue historique, 1934, p. 27.

30. Edward H. Carr, Qu'est-ce que l'histoire?, La Découverte, 1988, p. 31.

31. Dieter Wuckel, Science-fiction, une histoire illustrée, Leipzig, Éd. Leipzig, 1988, p. 224 sqq.; Jacques Van Herp, Panorama de la science-fiction, rééd., Bruxelles, Lefrancq, 1996, p. 293; Alexis Lecaye, Les Pirates du paradis. Essai sur la science-fiction, DenoëlGonthier, 1981, p. 149.

32. Lettre à L. Kugelmann, 17 mars 1871, dans Correspondance de Marx et Engels, Moscou, Éd. du Progrès, 1971, cit. entre autres dans Edward H. Carr, Qu'est-ce que l'histoire?, p. 161.

33. Charles Renouvier, Uchronie, rééd., Fayard, 1988. Sur l'uchronie en général, cf. Éric B. Henriet, L'Uchronie, Klincksieck, 2009.

34. Cf. Patrick Garcia, Jean Leduc, L'Enseignement de l'histoire en France de l'Ancien Régime à nos jours, Armand Colin, 2003, p. 262; Jean Stengers, Vertige de l'historien..., p. 64. 
de reconstituer par la pensée les possibles qui ne sont pas réels, bien que la plupart des historiens, et nous tous d'ailleurs, ayons tendance à croire que le passé a été fatal, et que l'avenir est indéterminé. Or le passé a été l'avenir des acteurs; il ne devient fatal qu'au seul sens où il a été et ne peut plus être changé. Mais il n'était pas fatal avant qu'il ne devienne réel: permettre de prendre conscience de l'homogénéité fondamentale entre le passé qui a été vécu et l'avenir qui reste à vivre, c'est probablement la fonction essentielle de cette histoire $[\ldots]^{35}$ ». Avec ces considérations, on quitte sans doute l'histoire stricto sensu, mais la façon d'écrire et de penser celle-ci est trop conditionnée par la vision générale du monde et de la causalité qu'a chacun pour qu'on puisse vraiment en faire l'économie... Peut-être est-ce d'ailleurs là le véritable apport de la counterfactual history tout comme de l'uchronie littéraire du point de vue de l'histoire. 